\title{
Coincidence Avoidance and Formulating the Access Problem
}

\author{
Sharon E. Berry <seberry@invariant.org >
}

In this paper, I discuss a trivialization worry for Hartry Field's official formulation of the access problem for mathematical realists, which was pointed out by $\varnothing$ ystein Linnebo (and has recently been made much of by Justin Clarke-Doane). I argue that various attempted reformulations of the Benacerraf problem fail to block trivialization, but that access worriers can better defend themselves by sticking closer to Hartry Field's initial informal characterization of the access problem in terms of (something like) general epistemic norms of coincidence avoidance.

\section{Introduction}

In "Mathematical Truth" (1973), Benacerraf presents a dilemma which includes the following classic worry for realists about mathematical objects - what is sometimes called the access problem. He argues that a certain causal constraint on knowledge, together with mathematical realism, implies that human knowledge of mathematics would be impossible. Many philosophers have been deeply moved by something about this worry (and analogous concerns in related domains), even while rejecting the specific premises employed in Benacerraf's argument (Field 1980; Linnebo 2006). However, a satisfactory formulation of this access worry has proved elusive.

In the first half of this paper, l'Il review Hartry Field's informal characterization of the access problem as arising from realists' (apparent) commitment to a match between human beliefs and realist facts, which cries out for explanation, and their (apparent) inability to provide such an explanation. I'll then discuss various attempts (by Field and others) to elaborate on this core idea by fixing on a single fact about human accuracy/reliability, $R$, which the mathematical realist must explain. I will note that these formulations face a trivialization problem (pointed out by $\varnothing$ ystein Linnebo and recently emphasized by the work of Justin Clarke-Doane and David Enoch) ${ }^{1}$ involving the apparent existence of explanations for the (supposedly explanation requiring) fact $R$ which do nothing to address intuitive access worries. And I

${ }^{1}$ Clarke-Doane argues that access worries should be rejected because they can't be satisfactorily formulated in a way that doesn't allow for trivializing response (2017). In contrast Enoch (2010) takes access worries seriously but argues that certain apparently trivializing responses can answer it. 
will argue that existing attempts to solve this trivialization problem (by changing or clarifying the fact $R$ to be explained) fail.

In the second half of this paper, l'Il advocate sticking much more closely to Field's original informal proposal when characterizing the access problem. Specifically, I'll argue that access worriers can reasonably state their concern (and reduce their confidence in realism until a solution can be found), without attempting to "cash this worry out" in various ways which have been presumed to be necessary in the literature. Specifically, access worriers needn't (and shouldn't) identify the access worry with a mere demand to coherently explain some reliability fact $R$. They also don't need to provide a nontrivial conceptual analysis of the notion of coincidence or an uncontroversially applicable criterion for being a coincidence.

Instead, they should simply formulate the access problem as follows. A realist theory of some domain (such as mathematics or morals) faces an access problem to the extent that adopting this theory² would require positing some "extra" coincidence 3 (about the match between human beliefs and reality), beyond those required by competing, less realist, approaches to the same domain. This unambitious formulation articulates the part of Field's proposal which almost everyone accepts, while avoiding the extraneous philosophical commitments (e.g., to a causal theory of reference, or a particular analysis of coincidence, or a single explanandum at issue in access worries) which have bedeviled previous proposals.

Understanding the access problem in this way (i.e., via direct appeal to notions of coincidence and coincidence avoidance) has some other important advantages. For example, it lets us attractively explain philosophers' failure to find a single reliability claim $R$ such that explaining $R$ suffices to banish access worries. And it clarifies what goes wrong with certain intuitively unsatisfying trivializing responses to access worries, which explain away one apparent coincidence involving human accuracy by appealing

2More specifically, adopting this claim together with typical claims about the extent of human knowledge regarding this domain seems to require positing some such coincidence.

3Some might argue that not all coincidences cry out for explanation, and only the latter tell against a theory in the way that gives force to an access worry. I'm not sure if that is correct, but I take no position on this issue here. For the sake of brevity, I will continue to talk simply about coincidences with the understanding that I mean coincidences which cry out for explanation. 
to another.

I will conclude by responding to some objections. First, one might object that we need to go beyond my unambitious formulation of the access problem if we hope to resolve philosophical disputes over the access problem. However, I argue that we can charitably state, and even plausibly hope to solve, disputes about the access problem in philosophy of mathematics without providing a further conceptual analysis of the access problem (or even the relevant notion of coincidence).

Second, one might suggest (as Clarke-Doane (2017) appears to) that intuitive dissatisfaction with trivializing explanations of human accuracy about realist mathematics and morals shows that our coincidence avoidance intuitions become unreliable when applied to theories involving necessary truths. But I argue that this principle should be rejected because accepting it would require junking an important and apparently fruitful part of current mathematical practice.

\section{Field's formulation of the access problem and trivialization worries}

\section{2.a Field's formulation of the access problem}

Let me begin by reviewing Hartry Field's approach to the access problem, and the trivialization worries which have arisen for it.

In Realism, Mathematics and Modality (1989), Field suggests that we should think of the access problem for mathematical realists as arising from a challenge for the realist to "explain how our beliefs about [mathematical objects] can so well reflect the facts about them" in some internally coherent fashion. He notes that, "[l]f it appears in principle impossible to explain this [match between our beliefs

and reality], then that tends to undermine ... belief in mathematical entities, despite whatever reason we might have for believing in them." I will develop and defend this core proposal in what follows.

However, Field elaborates this core idea in a way that (I will suggest) raises concerns about triviality. He argues that realists are committed to holding that, "for most mathematical sentences" $\phi$ the following reliability $R$ claim holds (we will discuss other ways of understanding this reliability claim below), and that some explanation for the truth of $R$ must be possible.

$R$ : Reliably, if mathematicians accept that " $\phi$," then $\phi$. 
Typical mathematical realists seem committed to accepting the above reliability claim. ${ }^{4}$ But, Field suggests, it appears in principle impossible for the realist to give any satisfactory explanation for $R$. And this fact casts doubt on the truth of realism.

This account of the access problem has obvious appeal. It has been used (with some minor modifications) to articulate access worries concerning other domains like morals and metaphysical possibility. ${ }^{5}$ Unlike Benacerraf's original access worry, Field's formulation does not depend on any contentious assumptions about causal constraints on knowledge. Furthermore, Field's formulation appears to reveal an internal tension within the (typical) realist's total web of beliefs. It thereby vindicates the common intuition that access worries are different from (and more troubling than) mere skepticism6.

I think Field is quite right that the mathematical realist faces strong epistemic pressure to explain $R$, and that dispelling the impression that they can't do so is a necessary condition for dissolving access worries. However, we will see that explaining $R$ is plausibly not sufficient to answer access worries. So

${ }^{4}$ Field (1989) writes that the Platonist's commitment to accepting this reliability claim is "beyond serious question." And Linnebo (2006) buttresses this idea by appealing to a connection between reliability and knowledge as follows. Admittedly, a thinker could have significant true mathematical beliefs without this kind of reliability. However, such a person would not qualify as having knowledge. For example, a "lucky fool" who decides whether or not to believe mathematical statements on the basis of a coin toss, and winds up with many true beliefs in this way, would (plausibly) not count as knowing these mathematical statements. However, the realist does take us to have knowledge. So they are committed to the stronger claim that we have reliable true belief, unlike the lucky fool.

5Just as it seems mysterious that our mathematical intuitions match objective facts about (say) platonic mathematical objects or proof transcendent coherence facts, it can seem mysterious that our a priori intuitions about goodness, beauty, or what Lewissian possible worlds exist match objective facts. 6It's not just that the access worrier can't justify their mathematical beliefs from indubitable premises which the skeptic accepts, but that their account of human accuracy seems troubling from their point of view. Also, note that the Fieldian access problem seems to point out a tension within the (typical) moral realist's total web of beliefs (including, e.g., various uncontroversial scientific and historical claims, and the idea that we have many true beliefs about moral topics), not within moral realism itself. A realist could (in principle) avoid Field's access problem by denying that we have any true moral beliefs or knowledge, but this fact provides little comfort to any actual moral realists. 
although Field's core approach is right and his further argument highlights crucial issues, it would be a mistake to take the final step of identifying access worries with an inability to explain $R$.

\section{2.b Safety and the trivialization problem}

To see why explaining $R$ (the fact that "Reliably, if mathematicians accept that ' $\phi$,' then ") seems insufficient to answer access worries, let's consider a few different ways of cashing this claim out.

One popular approach (Clarke-Doane 2014, 2017) is to read $R$ as demanding that our mathematical beliefs be "safe" in the sense that they could not have easily been wrong, i.e., mathematicians' belief-forming methods would not have lead them to form false beliefs at any suitably close possible worlds.

$R_{\text {safety }}$ In all sufficiently close possible worlds if mathematicians believe that $\phi$ then $\phi$

Another possibility, which Field mentions as a fallback option, is to drop the appeal to reliability and simply say that the actual abundance of true mathematical beliefs and lack of false mathematical beliefs is something for which the realist owes us an explanation.

But if one takes either of these approaches, then (as Øystein Linnebo [2006] and Justin ClarkeDoane [2017] have separately noted) it seems like one can "trivially" explain the relevant form of reliability using other premises which the realist accepts, as follows:

TRIV: Mathematicians reliably believe truths because they reliably believe only those mathematical claims which can be validly derived from a certain collection of mathematical 
necessary truths $\Sigma^{7}$

$T R I V$ seems to explain the safety of our mathematical beliefs. For, as it's robustly the case that mathematicians form mathematical beliefs entailed by $\Sigma$, they will continue to form mathematical beliefs entailed by $\Sigma$ in all relevantly close possible worlds. And all propositions entailed by propositions in $\Sigma$ are necessary truths. Hence all these close possible worlds will be ones in which they continue to form mostly true mathematical beliefs, thereby explaining the safety of our mathematical beliefs. One might think of TRIV as explaining safety via the fact that if our mathematical methods are accurate, then those methods are necessarily so. And TRIV also seems to explain (at least in some sense) our possession of many true and few false mathematical beliefs (in the actual world), by pointing out that we arrive at mathematical beliefs by reasoning validly from true axioms.

However, it is equally clear that citing TRIV does nothing to assuage intuitive access worries. This suggests that intuitive access worries cannot be reduced to the need to explain either the safety of our mathematical beliefs or the fact that we have many true and few false mathematical beliefs.

Now Field could, obviously, respond to this objection by denying that TRIV constitutes a genuine explanation for $R$ (or for our possession of many true and few false mathematical beliefs). And this idea has some prima facie attraction.

7One might object that this version of Linnebo's trivializing explanation doesn't account for our true belief in the consistency of ZFC (or in the arithmetical sentence $\operatorname{CON}(Z F C)$ ). However, I think one can naturally extend the trivializing explanation to explain our true belief in $\operatorname{Con}(Z F C)$ as follows. If we only need to explain $R$ for most mathematical claims encountered in normal mathematical practice, it suffices to let $\Sigma$ consist of $Z F C$ plus all finite iterates of the $C O N$ operator (i.e., ZFC $+C O N(Z F C)+C O N(Z F C$ $+\operatorname{CON}(Z F C)) \ldots$. .). Of course, it is probably true that, for some computable ordinals $\alpha$, we believe $\operatorname{CON}^{\alpha}(Z F C)$ (where $\operatorname{CON}^{\alpha}$ indicates iterating the $\operatorname{CON}$ operator $\alpha$ many times). However, our inability to know which putative computable ordinals are truly well ordered prevents this chain from continuing indefinitely. So one can give a similar explanation for our accuracy about even claims derivable from these infinitary iterates of the CON operator. Namely, there is some computable ordinal $\beta$ (though not one who we recognize any description of as a computable ordinal) such that the $\beta$-iteration of $C O N$ applied to ZFC is both true and entails all the iterated CON sentences we accept. 
However, many readers (like Linnebo and Clarke-Doane) seem to have the opposite intuition. And I think it is ultimately hard to deny that TRIV provides some kind of an explanation of $R$. For we can easily imagine nonphilosophical contexts where TRIV would constitute an excellent response to an explanatory demand: an anthropologist could explain why some newly discovered community is reliable about mathematics/ that is explain why the community reliably had so many true and so few false mathematical beliefs by showing that all their mathematical reasoning can be reconstructed in terms of some formal system and then noting that this system is sound. ${ }^{8}$ So, in the absence of further sharpening of the intuitive notion of explanation (something Field doesn't provide), it appears that TRIV does explain $R$ and access worries cannot be reduced to the need to explain $R$.

Note, also, that one cannot defend Field's account of the access problem by rejecting TRIV merely on the grounds that it assumes the theorems of $\Sigma$ are true in a realist sense (e.g., correctly describe the platonic objects) which philosophers pressing an access worry wouldn't accept because this doesn't prevent TRIV from being an internally coherent explanation from the realist's perspective of our accuracy about mathematics. One of the great benefits of Field's proposal was that it appeared to reveal an internal problem for realism, not just a skeptical worry. Thus, it suffices for the realist to give an internally coherent explanation.

\section{2.c Interpreting R more demandingly}

One natural thought is to interpret the "reliability" invoked in Field's $R$ more demandingly, and use this as a basis for rejecting TRIV.

Suppose we grant that TRIV explains why there aren't any extremely close possible words at which mathematicians' beliefs are massively false. If we read Field's reliability claim $R$ more demandingly -as requiring mathematicians to be accurate in a larger sphere of close possible words including somewhere they don't form beliefs via $\Sigma$-then we can still resist the claim that TRIV explains $R .{ }^{9}$

It's not immediately obvious that the realist is committed to the truth of such a demanding

8Such explanation would admittedly be partial, but that doesn't prevent it from being an explanation. As David Lewis (1986) notes, everything we give is a partial explanation: the accident occurred because of the bald tire, because of the driver's slipshod maintenance, etc.

9This corresponds to individuating our methods more broadly. 
version of $R$. Rigorously defending this approach would require arguing that the realist is committed to some specific and much higher degree of reliability, and I haven't seen anyone do this. ${ }^{10}$

But I won't dwell on this hurdle, as I think a deeper problem is lurking. The problem is that we can imagine discoveries which would imply and (in a sense) explain even very modally robust agreement between human psychology and realist facts about something like math or morals, while still leaving intuitive access worries untouched. Thus, a more demanding interpretation of $R$ is incapable of rescuing Field's elaboration of his core intuitions.

For example, consider the classic moral realist, who takes our beliefs about permissible favoritism toward relatives to be "robustly objectively correct" in a sense which implies that creatures apparently inclined to advocate and practice a different degree of favoritism would have false beliefs about morality (rather than true beliefs about some other notion "shmorality" of equal metaphysical status). Moral realists of this stripe intuitively face an access worry about the accuracy of our moral beliefs. ${ }^{11}$ Now imagine such a moral realist attempting to address access worries by giving the following kind of explanation of our accuracy about permissible favoritism facts.

EV-MOR: It is a robust fact that in all circumstances conducive to the evolution of intelligence, natural selection favors the trait of advocating and valuing as being twice as generous with immediate family as with other individuals. Furthermore, it is morally correct to be (exactly) twice as generous with family, and this is a necessary truth.

This story certainly seems to provide some kind of explanation for our accuracy about moral facts in a very wide range of possible worlds, yet considering it does nothing to answer intuitive access

10This is a version of the famous "generality problem" for reliablist epistemologies (Goldman and Beddor, 2016).

${ }^{11}$ Note that even imperfect moral accuracy (at a rate substantially better than chance) can give rise to such an access worry. 
worries. ${ }^{12}$ This is not just because the genealogy of morals suggested above is probably false. For even if we imagine that the evolutionary/game theory part of EV-MOR were unquestionably true and getting at a deeply reliable law of nature, considering EV-MOR would still do nothing to address intuitive access worries. Thus, Field's official formulation of the access problem can't be rescued by increasing the level of reliability (in the sense of safety) which is to be explained.

\section{2.d Sensitivity and counter-possible conditionals}

A different strategy for understanding the reliability claim in Field's $R$ is to appeal to metaphysically impossible worlds.

Employing metaphysically impossible worlds has little effect on safety. ${ }^{13}$ However, it does give teeth to sensitivity requirements (another popular way of thinking about reliability). Sensitivity demands that if $\phi$ hadn't been true, we wouldn't have believed $\phi$ (i.e., in the closest possible worlds where $\phi$ isn't true, we don't believe $\phi$ ). Our mathematical beliefs are trivially sensitive if we interpret this requirement using regular Lewisian counterfactuals (because there are no possible worlds where they are false).

However, demanding that realists explain sensitivity at metaphysically impossible worlds promises to let us reject explanations like TRIV and EV-MOR. For the fact that mathematicians reliably tend to accept propositions derivable from certain necessarily true axioms doesn't appear to explain why, in metaphysically impossible worlds where these axioms are false, we would still wind up having true

${ }^{12} \mathrm{~A}$ similarly unsatisfying example explanation can be developed in the case of mathematics.

EV-MATH: The only way for intelligence to evolve involves having a compositional language, and the only way that mathematics-like practices ever arise involves fluke reusing the brain structures which compute grammaticality to produce assertions about certain mathematical structures, and it just so happens that these correspond to the platonic mathematical objects which actually exist.

${ }^{13}$ As Justin Clarke-Doane points out, even if we allow that "impossible worlds" where mathematical facts are different can in principle be relevant to truth conditions for counterfactuals, it would seem that these worlds would be very remote from the actual one. So it's not clear why explaining reliability should require showing that mathematicians' beliefs would continue to express truths in these very remote possible worlds (2017). 
mathematical beliefs. Indeed, such explanations seem to suggest that if mathematics/morals had been different, then our beliefs would have been just the same (because these beliefs are shaped by unrelated evolutionary/game-theoretic/anatomical considerations).

However, this approach faces very serious problems. First, there are reasons for doubting that we have any coherent shared grip on the closeness relation for metaphysically impossible scenarios (aka "counterpossible conditionals"). For example, if $2+2=5$ would + still satisfy the usual inductive definition? If not, how would things be different? Despite advances in understanding the logic of counterpossible conditionals (Nolan 1997), we still face significant uncertainty (or perhaps conceptual underdetermination) concerning the substantive closeness relation on impossible worlds. ${ }^{14}$ Given this uncertainty, cashing out informal access worries in terms of a demand to explain counterpossible conditionals doesn't seem very helpful.

A second problem for this approach is that the counter-possible sensitivity requirement seems to fail (or counter-possible sensitivity seems hard to explain) in many cases which are intuitively unproblematic. For example, if bachelors were unmarred women rather than unmarried men, would we still believe that bachelors are unmarried men?15 Presumably, there is no reason to doubt our knowledge of bachelorhood facts, and this calls into question this interpretation of the sensitivity requirement above. ${ }^{16}$

${ }^{14} \mathrm{Or}$ the substantive closeness relation which would be relevant to this attempt to formulate access worries, if there is some kind of context dependence as David Lewis has suggested (1986b).

15Justin Clarke-Doane (2017) gives a somewhat more complicated example along these lines: If the facts about what configurations of matter constituted a chair were different, would our beliefs be different? ${ }^{16} \mathrm{~A}$ third problem for cashing out the access worrier's demand in terms of any sensitivity demand is pointed out by Donaldson (2014). Imagine someone who forms the belief that none of her colleagues' lottery tickets will win based merely on the fact that there are a million other tickets in some lottery and only one that will win. Her beliefs may well not be sensitive: had one of her colleagues won the lottery she would have still expected them to lose. Yet her accuracy will not be mysterious or coincidental or give rise to any kind of intuitive access problem. Thus, explaining human accuracy in Field's sense should not require sensitivity. 


\section{Linnebo and alternative languages}

Now let us turn to a variant on Field's $R$ suggested by $\varnothing$ ystein Linnebo (2006). Linnebo discusses a version of the problem for cashing out $R$ in terms of sensitivity noted above. He then highlights a different kind of “counterfactual dependence of people's disposition to accept mathematical sentences upon those sentences being true" (566), which might be relevant to access worries.

Specifically, he proposes that a good strategy for answering access worries could involve defending a metasemantic claim along the following lines.

$R_{M S}$ If mathematical sentences (like " $2+2=4$ ") had not expressed truths, then mathematicians wouldn't have accepted them.

In terms of possible worlds, $R_{M S}$ asserts that the closest possible worlds in which linguistic differences ensure that the sentence " $2+2=4$ " expresses a falsehood are ones in which mathematicians no longer accept this sentence. ${ }^{17}$ Thus, we can think of $R_{M S}$ as spelling out the sensitivity requirement from the prior section using counterfactuals about semantic facts instead of metaphysically impossible worlds to spell out the sensitivity requirement from the prior section.

Now $R_{M S}$ might seem like a promising candidate for the reliability claim $R$ in Field's formal proposal. ${ }^{18}$ For, intuitively, TRIV seems bad because the connection between the two sides of the explanandum look fortuitous. In many cases, one can distinguish this kind of fortuitous agreement by looking to counterfactual sensitivity. But, as we have seen, a straightforward counterfactual sensitivity analysis runs into problems with metaphysically necessary claims. Thus, one might be inclined to turn to

\footnotetext{
17So, for example, mathematicians in this world don't assent to this sentence in conversation or place it into textbooks.

${ }^{18}$ Linnebo does not commit himself to this claim. He merely suggests that $a$ good answer to access worries could take the form of an explanation for $R_{M S}$, not that any explanation for why $R_{M S}$ is true would suffice to answer access worries.
} 
Linnebo's linguistic counterfactual for a more satisfactory account.

However, Linnebo's counterfactual faces its own trivialization problem as well as an overdemandingness problem. The trivialization worry arises as follows. ${ }^{19}$ It's hard to be confident about what the closest possible words at which " $2+2=4$ " doesn't express a truth look like-something which might already be cited as an inconvenient aspect of Linnebo's view. But to the extent that I grasp this notion at all, it seems it might well be that the closest possible worlds where " $2+2=4$ " doesn't express a truth are ones where some superficial and recent change in language/orthography went differently. However, we can explain why mathematicians at these worlds don't accept " $2+2=4$ " in an intuitively unsatisfying fashion just by citing the principle that when linguistic/orthographic changes are made, people adjust what sentences they endorse accordingly.

For example, these closest possible worlds might well be ones where the transition from Roman numerals to Arabic numerals went differently so that the symbol " 2 " was used to mean " 3 in most of the western world, for note that the history of such worlds could be exactly like that of the actual world up to this orthographic change. And it seems imaginable that a rather small copying error (a Lewisian "minor miracle") propagated by a few monks at some key bottleneck in communication between the Arabic numeral and Roman numeral using mathematical communities could have produced such a difference in orthography (and, hence, in the meaning and truth value of the relevant sentence). ${ }^{20}$

So plausibly we can explain why we wouldn't have accepted " $2+2=4$ " in a world where "2" named "3" (in English) as follows. In these possible worlds, at the time that the transition to Arabic numerals occurred, speakers were reliably disposed to confidently reject sentences using Roman numerals to express the proposition $3+3=4$. Thus, the principle that (considered, confident) views aren't affected by changes in orthography explains why people in those worlds didn't accept " $2+2=4$ " immediately following the change in orthography, and simple inertia explains why later generations

19 am indebted to Warren Goldfarb for formative conversations on this point.

20I don't know how plausible it is that just few transcription errors of this type could have resulted in this difference. But I take that detail not to matter much for my argument because it would be bad enough if Linnebo's formulation implied that had our choice of symbols (or words) been so highly contingent there would be no access problem or access worries would be trivially solvable. 
continued to reject them.

Putting this together gives us the following explanation for $R_{M S}$. Plausibly, the closest possible worlds where " $2+2=4$ " expresses a falsehood are ones where this is so just because of some change in orthography (e.g., where " 2 " is adopted as the name for " 3 " instead of 2 , so " $2+2=4$ " expresses the mathematical falsehood " $3+3=4$ "). But such changes in orthography don't tend to change what propositions people accept. ${ }^{21}$ So, given that people were disposed to reject $3+3=4$ when working with Roman numerals, they'd likely continue to reject it after adopting (this modified version of the) Arabic numerals and intellectual inertia could explain why later generations would continue to reject it.22 This explanation is unsatisfying because it explains peoples' accuracy about mathematics at a later time simply by appeal to their accuracy about mathematics at an earlier time, plus a principle of continuity regarding their beliefs.

More generally, Linnebo's $R_{M S}$ conditional seems to be potentially explicable via the "deeper," unsatisfying explanations for human accuracy about mathematical/moral facts discussed in the previous section. For instance, EV-MOR asserted that evolution and game theory determine that intelligent creatures are overwhelmingly likely to treat a certain amount of favoritism as permissible, and that ratio of permissible favoritism also happens to be objectively correct. Now imagine discovering that evolutionary and psychological mechanisms gave us moral sentiments matching this game-theoretic ideal in a way that was very counterfactually robust. So, for example, smallish changes to the human evolutionary environment would have made little difference to the moral sentiments with which we

${ }^{21}$ That is, people identify what sentences in the new system correspond to the sentences they accepted in the old orthography and accept those in the new orthography.

22One might worry that because we often do change beliefs when they turn out to produce practically harmful results, the person responding to (this version of) the access problem is on the hook to explain why rejecting the sentence " $2+2=4$ " doesn't cause harmful outcomes. However, one can respond to this concern by extending the explanation to include the fact that rejecting the sentence " $3+3=4$ " doesn't seem to lead to practical difficulties in the actual world and arguing that the similar inferential role played by " $2+2=4$ " in a world with the orthographic change in question suffices to explain the lack of practical difficulties as a result of rejecting " $2+2=4$ " and, thus, explain why it would continue to be rejected in such a world. 
wound up. And a human raised in almost any environment where they could learn to talk, survive to adulthood, etc. would be very likely to form some concept with the action-guiding role which we assign to permissibility and have similar intuitions to the ones we do about how this concept applies.

Learning that our moral sentiments were robust in this way would make it very plausible that, if language had been different (so other moral sentences had expressed truths), we would still have been disposed to accept the same moral propositions (and hence, from the realist point of view) still accepted true moral propositions. Thus, it would provide a direct explanation for Linnebo's counterfactual. But it would do nothing to assuage access worries. Assuming EV-MOR is true, the closest worlds where "helping friends twice as much as strangers is permissible" expresses a falsehood would plausibly be ones where our language is different (rather than our moral sentiments) so that we don't accept this sentence (and Linnebo's metasemantic variant on the sensitivity requirement is satisfied). Thus, if EVMOR were true, it would plausibly explain $R_{M S}$ (as well as Field's $R$ ) without answering intuitive access worries.

This caveat raises the issue of what kind of grip we have on these linguistic counterfactuals at all. For example, if "there are dogs" had expressed a falsehood, what claim would it have expressed? Would it still have expressed a true claim? There are many different scenarios where some sequence of symbols like " $2+2=4$ " fails to express a truth and it's not at all clear that the closest such worlds are ones in which " $2+2=4$ " even has anything to do with mathematics. This brings us to a second problem.

The problem is that we can construct cases where some quirk of history ensures the falsehood of the counterfactual $R_{M S}$ in a way that does nothing to generate an intuitive access worry or any kind of problem with positing knowledge. For example, it's been argued that medieval science often expected deep analogies between different domains, so that very different things (personality types, metals, planets, mythical Greek gods) which somehow participated in the nature of Neptune would behave analogously. Imagine a possible world where analogous theories were developed for astrology and fledgling chemistry (and each had a special notation) such that there was a fairly simple correspondence between sentences expressing (supposed) truths of the astrological theory and those expressing (supposed) truths of the chemical theory in the year 800 CE. Now suppose that because of these 
analogies, some monastic copying error swapped the symbols used to express chemical reactions and astrological claims so that " $\mathrm{H}^{+}+\mathrm{OH}^{-} \leftrightharpoons \mathrm{H}_{2} \mathrm{O}^{\prime}$ went from originally expressing an astrological claim (say, the proposition that male Leos and female Libras are romantically linked when Mars is entering Scorpio) to expressing the claim that it expresses in normal English. And suppose that chemistry and astrology developed separately in the years after 800 , with both continuing to enjoy great popularity. We can imagine a chemist who has (intuitively) justified beliefs about chemistry and unjustified beliefs about astrology. Plausibly, some of the closest possible worlds to this one where " $\mathrm{H}^{+}+\mathrm{OH}^{-} \leftrightharpoons \mathrm{H}_{2} \mathrm{O}^{\prime \prime}$ fails to express a truth would be ones where this copying error never happened (rather than the very remote ones in which the chemical reactions proceed differently). In such worlds, the above sentence will express a widespread and a long-standing, but false, doctrine about astronomy, which our horoscopereading chemist also accepts. Thus, it won't be the case that had various chemical sentences not expressed a truth, she wouldn't have believed them. Yet intuitively our chemist could qualify as having chemical propositions actually expressed by these sentences. Thus, we seem to have a counterexample to $R_{M S}$.

This final problem is only heightened if we try to avoid trivializing explanations (like the Roman numerals example discussed above) by strengthening our reliability requirements. For doing this only increases the risk of demanding too much, i.e., that Linnebo's conditional $R_{M S}$ could fail for reasons (like the chancy chemical-astrological symbol swap) that do nothing to impugn our claims to knowledge of a given domain. Thus, there's no plausible interpretation of Linnebo's $R_{M S}$ which lets us avoid both trivialization worries and appeal to a sensitivity principle which we have independent reason for doubting.

Stepping back for a moment, I think the core problem for this proposal is the same one that generates trivializing answers to the other formulations of the access problem above. The realist can almost always explain a given fact $R$ about human mathematical accuracy if they are allowed to assume -and use unexplained-every other fact about the match between human psychology and objective mathematical reality which they believe in. But such explanations won't satisfy access worriers because 
doing this amounts to showing that the existence of one prima facie mysterious match between human psychology and objective mathematical fact is unsurprising given the existence of another such match. In the current case, it seems that no explanation which brutely appeals to the fact that people got mathematics right at some earlier time, e.g., when we were using Roman numerals, cuts ice with regard to assuaging intuitive access worries. Yet invoking such facts seems quite relevant and useful in explaining why the closest possible worlds where " $2+2=4$ " expresses a different (false) proposition are ones in which we don't accept " $2+2=4$."

I read philosophers like Clarke-Doane as, in effect, suggesting that such trivializing explanations pose a dilemma for the access worrier. Either the access worrier abandons Field's ambition of locating a tension within the mathematical realist's own worldview ${ }^{23}$ or they allow the realist to explain one seemingly mysterious match between human psychology and objective mathematical facts which they believe in by appeal to another (since belief in these other apparent coincidences is, after all, part of the mathematical realist's worldview). Thus, it might seem that the access problem is, ultimately, an illusion. However, we can tidily avoid both horns of the dilemma by rejecting the hidden premise that access worries are simply a matter of realists' inability to explain some reliability fact $R$. Below I will argue for the following picture (which we get by taking Field's informal version of the access problem seriously). Access Problems aren't a matter of realists' inability to provide any explanation for some fact. Instead, they arise from the interaction between realists' intuitions about what kinds of explanations certain facts cry out for (i.e., intuitions about coincidence which they share with antirealists) and the (disappointing) nature of the explanations the realist can provide.

\footnotetext{
23If the access worrier does this, they can reject trivializing explanations as employing premises which beg the question against skeptics about mathematical realism like themselves. But this comes at a very serious cost. For it's no longer clear that they have located a problem for mathematical realism, as opposed to merely showing the possibility for internally coherent doubt about some portion of the things which the mathematical realists believes, i.e., merely showing that mathematical realism is not indubitable (something nearly all contemporary philosophers would be happy to grant since the doctrine that knowledge requires indubitability is widely rejected).
} 


\section{A coincidence avoidance approach to access worries}

\section{4.a Field's core idea and general norms of coincidence avoidance}

In view of the problems for spelling out (or replacing) Field's explanandum $R$ discussed above, I propose that we stick to Field's initial characterization of the access problem in terms of general norms of coincidence avoidance, rather than trying to specify any single reliability fact, such that merely explaining this fact (from more general premises which the realist believes) would suffice to answer access worries.

We should, instead, simply say something like the following. A realist theory of some domain of investigation (such as mathematics or morals) faces an access problem to the extent that accepting it commits one to positing a certain kind of coincidental match between human beliefs and the facts about that domain, but prevents one from giving any explanation which would remove this appearance of coincidence. A little more formally, a realist theory faces an access problem to the extent that:

Combining this theory with uncontroversial claims about the extent of human accuracy about the domain in question forces us to posit some coincidental match between human beliefs and belief-independent facts (a match which intuitively "cries out for explanation," but has no explanation).

When this holds, it would seem that we have a significant (if defeasible) reason to reject the realist theory in question. Such theories are ceterus paribus undesirable in that they commit us to positing an extra inexplicable coincidence: a match between human psychology and the realist's subject matter, which cries out for explanation but cannot be explained.

Note that this constitutes an internal problem for advocates of the relevant realist theory. For the shared norms of coincidence avoidance which we draw on in phrasing access worries are themselves part of the realist's total picture of reality. Thus, (we can continue to say that) the realist faces an internal tension-in this case, a tension between their philosophical beliefs about some domain and their own 
sense of which kinds of correlations constitute an unattractive coincidence. ${ }^{24}$ Also note that, on the view I'm advocating, access worries only give us ceterus paribus reason to reject a given realist theory of some domain. If it turns out that all the alternative views which avoid this access problem have worse flaws (as, e.g., formalist theories which have trouble capturing proof transcendent truth conditions and the role of math in the sciences plausibly do), this bullet might be worth biting.

While, strictly speaking, a theory has an access problem to the extent no satisfactory explanation of the match between beliefs and belief-independent facts is possible, we can sometimes also speak loosely and say that a theory faces an access problem when it appears that no such explanation is possible (though, to be pedantic, it only apparently faces an access problem). When it no longer appears that no such satisfactory explanation is possible, we would say that the (apparent) access problem has been solved or dissolved. Thus, classical attempts to eliminate access worries like Modal-Structuralism, Quantifier Variance, Quineinism, and Neo-Fregean view can be seen as attempts to solve (or partially solve) the access problem as conceptualized above. ${ }^{25}$

\section{4.b Helpful consequences}

Formulating Field's access worry as an application of more general norms of coincidence avoidance has two interesting and helpful consequences.

First, this proposal identifies access worries with a holistic problem with the realist's account and thus explains why (as noted above) they can't be dismissed by explaining one type of accuracy in terms of another, equally mysterious, type of accuracy.

${ }^{24}$ While philosophers like Clarke-Doane represent access worries as presenting new evidence, I think they are-like mathematical arguments-making an a priori philosophical point (hence presenting facts which they think an ideal Bayesian agent would already have recognized rather than presenting new evidence on which such an agent would update). This difference may also help explain the different conclusions we reach about intuitively unsatisfying explanations like TRIV and EV-MOR.

25Modal-Structuralism (Hellman 1994), Quantifier Variance (Hirsch 2010), and Neo-Fregeanism (Wright 1983) help answer access worries (as characterized above) by suggesting that (almost) any logically coherent mathematical posits would express truths and thus explaining how any coherent mathematical beliefs we have correspond to mathematical truths; of course, the issue of how we come to have coherent mathematical beliefs remains. 
For, on the view above, (dis)solving one's access problem requires removing the appearance that one is committed to positing any extra coincidences. So we can allow that TRIV and EV-MOR do, in some sense, explain human possession of true beliefs but still maintain that they are useless in addressing access worries because each makes salient appeal to an extra coincidence, which more deflationary rival understandings of mathematical/moral practice let us avoid. Specifically, TRIV explains our accuracy about realist mathematical facts by appealing to an unexplained coincidental-seeming match between our mathematical reasoning method (our acceptance of sentences in $\Sigma$ as something like mathematical axioms) and realist mathematical facts. And EV-MOR only explains our good intuitions about morality by appealing to an unexplained match between game-theoretic optimality and objective moral facts.

Second, this approach suggests an important way in which access worries can be a matter of degree. While a philosophical theory either does or doesn't allow for an explanation of $R$ or $R_{M S}$ (and thus does or doesn't face an access problem), on this approach, one theory can be preferred to another as it requires accepting fewer coincidences.

Because of this comparative element, we should not think of access worries as invoking an epistemic requirement to "consign to the flames" every theory that posits a coincidence (analogous to Hume's famous empiricist exhortation to reject all concepts that weren't suitably related to experience [2007)]). Instead, access worriers appeal to general norms in favor of reducing the number of coincidences one is committed to positing, insofar as this is compatible with other epistemic goals.

This is important and helpful because it means that, even if our knowledge of inductive generalization raises an access problem in its own right (maybe even an insoluble access problem), we can still invoke inductive generalization to dispel our access worries regarding a domain like mathematics (as no rival theory would dispel the coincidence that the future seems to behave like the past). Thus, theories can suffer access worries to varying degrees depending on the number and implausibility of the coincidences they are committed to positing.

\section{4.c Do we owe a further analysis of coincidence?}

This way of understanding access worries can seem to require using an unacceptably imprecise notion of coincidence avoidance. However, the same imprecise notion already plays an important role in scientific 
and philosophical reasoning.

We clearly have a practice of distinguishing certain parts of a theory as unattractive coincidences. And we take commitment to any such extra coincidences to be a (ceterus paribus) reason to disfavor a theory. Think of the kind of argument we might use to convince someone to stop believing in the Loch Ness monster. We generally wouldn't be able to derive the nonexistence of the monster from beliefs we share with the Loch Ness conspiracy theorist or locate a literal contradiction within their beliefs. Rather, we would point out unattractive extra coincidences that the Loch Ness monster theory has to admit (the monster never shows up when someone has a really good camera, it only appears in pictures which could plausibly be faked, etc.) but can't elegantly explain. We would appeal to a kind of shared general epistemic norm, which says that one has ceterus paribus reason to avoid theories which posit certain kinds of (inexplicable) coincidences. What results isn't a deduction that the Loch Ness monster doesn't exist, but, rather, ceterus paribus reasons for disfavoring its existence.

Admittedly, what makes something a coincidence is rather complicated. ${ }^{26}$ Coincidences aren't just facts posited by a theory which would otherwise be assigned low probability given the rest of a theory. For example, any particular long sequence of outcomes of a coin toss is unlikely, but we don't take total theories of the world which include the results of past coin tosses to be committed to an extra unattractive coincidence. ${ }^{27}$ Nonetheless, spotting and rejecting such coincidences plays an important role in scientific and commonsense reasoning, ${ }^{28}$ even when we can't appeal to anything like a general Carnapian logic of induction. We might wish to have a tidy and uncontroversial criterion for when a theory counts as positing extra coincidences. However, we are all committed to using this kind of reasoning all the time on a "know it when you see it" basis. Thus, it seems reasonable to take these intuitions about theoretical badness at face value.

26See Lando (2016) and Bhogal (Forthcoming) for some examples of recent work on this project. 27The feeling of coincidence/crying out for explanation seems related to an intuition that some other theory predicting the same things but with fewer dimensions of freedom should exist, but the question of a priori theory plausibility is an infamously hard one and I won't speculate about this more here. ${ }^{28}$ For example, the clustering of the orbits of many trans-Neptunian objects has lead astronomers to hypothesize the existence of a ninth planet orbiting beyond $200 \mathrm{AU}$ (Wikipedia 2016). 


\section{Objections}

\section{5.a Tractability}

Let me conclude by addressing two objections. The first objection concerns the tractability of disputes concerning access problems. Many philosophers currently disagree about how much of an access worry various forms of realism about mathematics, morals, etc. face.

In this paper, I have argued that critics of mathematical/moral realism can reasonably articulate and press an access worry by appealing to shared intuitive norms of coincidence avoidance while taking a "know it when we see it" attitude to the relevant concept of coincidence, rather than providing any explicit theory of what it takes for something to be an unattractive coincidence.

But one might fear that adopting this position makes disputes about the access problem deeply intractable by letting access worriers issue their challenge from an unassailable swampland of brute intuitions without committing themselves to any general theses which the realist could defend themselves by attacking.

However, I will argue that such pessimism is unwarranted because there are other credible ways in which debate about access worries can be carried out, and by which widespread philosophical agreement could plausibly be produced.

On one hand, realists can reasonably hope to win over opponents by providing a suitable sample explanation for our accuracy about the relevant domain which suffices to banish coincidences (or only employs coincidences which antirealists about the relevant domain are also committed to accepting). I propose such a story in "Not Companions in Guilt (2018) and "The Residual Access Problem (Forthcoming).

Conversely, there are also credible paths to philosophical agreement that there is a genuine access problem for realism about a given domain. For example, a history of massive effort and continued failure to discover any plausible explanation of a certain coincidence can itself gradually increase access worries on my account. Thus, this way of formulating the access problem provides a way for access worries to get worse and a way for them to get better. 


\section{5.b Coincidences involving necessary truths}

The second (and final) objection I want to consider concerns the reliability of our intuitions about coincidences and coincidence avoidance in domains involving necessary truths.

One might imagine the philosophers like Justin Clarke-Doane (2017) who have pressed trivializing responses to the access problem responding to my proposal as follows. ${ }^{29}$ They might allow the above general point about the general legitimacy and usefulness of coincidence-avoidance intuitions but suggest (perhaps partly on the basis of mathematical access worriers' failure to cash out their intuitive appeals to coincidence avoidance in other terms) that something special goes wrong when we apply these intuitions to evaluating whether mathematical realists face an access problem. Specifically, one can think of them as suggesting that (either) our coincidence-avoidance intuitions about which correlations involving necessary truths "cry out for explanation" are deeply unreliable, or that (appearances

29 Clarke-Doane (2017) proposes that access worries cannot call into doubt the safety or sensitivity of a realist's beliefs if the realist can explain the safety and sensitivity of her beliefs from other premises she accepts.

However, I would argue the mere fact that a web of belief contains elements that imply/explain the safety and sensitivity of some faculty/belief-forming mechanism, doesn't prevent this web of beliefs from having other features which call this safety and sensitivity into doubt. For example, I might have a great story (involving optics, brain processing, etc.) about how using my eyes and memory provided me with many safe and sensitive beliefs about Jane's office, so this aspect of my total picture of myself may look great. But if my other beliefs imply that the air in Jane's room contains a hallucination-inducing drug which would interfere with this belief-forming mechanisms, this will give me reason to doubt both the truth of my beliefs about Jane's room and their safety and sensitivity. My ability to provide a (so to speak) "locally" internally coherent explanation for how my beliefs about Jane's room are safe and sensitive doesn't mean that I shouldn't doubt this safety and sensitivity because, among other things, it doesn't imply that my total web of beliefs is free of tensions.

And, on the picture I have painted above, access worriers feel something similar is going on with the realist who explains her moral reliability via EV-MOR or her mathematical reliability via TRIV. The premises which the realist believes and uses in EV-MOR or TRIV provide a good explanation for our reliability about realist morals/mathematics if they are true. But this fact alone doesn't guarantee that other elements within her total web of beliefs (such as norms that we should minimize our commitment to positing certain kinds of inexplicable coincidence) can't give her reason to doubt these premises. 
notwithstanding) all such cries for explanation can be adequately answered by just by "stapling together" two unrelated explanations for each half of the coincidence (as these trivializing explanations do).

However, I think this line is hard to maintain. First, trivializers haven't presented much reason for thinking that analyzing the notion of coincidence avoidance in cases where both sides of the relevant coincidence are contingent truths is any easier. No substantive (informative) analysis of what it takes for a contingent regularity to cry out for explanation is widely accepted. And there are plenty of good paradigms for thinking about coincidence avoidance which apply equally to necessary and contingent regularities (e.g., one might relate coincidence avoidance to a preference for theories that have fewer degrees of freedom or a general scientific desideratum to favor theories that unify [Kitcher 1981]).

Second, and more importantly, saying that our intuitions about coincidence avoidance become incoherent when applied to necessary truths seems to conflict with existing mathematical methodology. For mathematicians seem to fruitfully use explanation seeking and coincidence avoidance intuitions (including the intuition that merely "stapling together" two unrelated, but modally robust, explanations for each half of an apparent coincidence is unsatisfactory) to guide research (Baker 2009; Lange 2010). The history of John Conway's "Monsterous Moonshine" conjecture provides a dramatic illustration of this. It shows how discovering a relationship between pure mathematical facts which intuitively "cries out for explanation," and, then, seeking such an explanation can lead to important discoveries even when a proof of both facts already exists.

In this episode, mathematicians noticed that the same number-196,883-appeared in two seemingly unconnected areas of mathematics. It appeared both as one of the dimensions of the monster group (the largest of the sporadic simple groups) and as the first nontrivial coefficient of the $j$-function (an important function in number theory). Later mathematicians discovered further that the second nontrivial coefficient of the $j$-function was the sum of the first three special dimensions of the monster group.

Despite the lack of prior reason to believe that there was any connection between these two areas of mathematics, the fact that these coincidences seemed to call out for explanation motivated mathematicians to hypothesize a connection and eventually discover one which lead to deep 
mathematical insights. Mathematicians thought there must be some further explanation for the above regularity involving necessary truths on both sides (and they turned out to be right) (Klarreich 2017).

So I think considering mathematical cases like the one above tells strongly against any suggestion that our intuitions about coincidence avoidance become (generally) unreliable when applied to necessary truths. Similarly, in philosophy, we seem happy to accept that avoiding coincidence in the sense of favoring theories that unify many explanations with few resources.

And proponents of trivializing explanation like Clarke-Doane haven't shown that there's any principled and theoretically attractive line which carves off the specific intuitions about coincidence avoidance and necessary truths which he wants us to be suspicious of (those driving access worries) from general methods of reasoning which are attractive and ubiquitous in philosophy and mathematics. Therefore, absent a stronger argument that such reasoning leads us astray, I don't see any reason to eschew its use. ${ }^{30}$

\section{Conclusion}

In this paper, I discussed a trivialization problem for Hartry Field's formal characterization of the access

30Now one might further ask: Is there any mathematical precedent/analog for the access worrier's overall suggestion that norms of coincidence avoidance should motivate us to reject an antecedently attractive metaethical theory, such as realism? Can recognizing a mathematical regularity's cries for an explanation ever make it rational to reject a previously attractive theory? (Thanks to an anonymous reviewer for suggesting this question.)

Perhaps Gödel's idea that new axioms for set theory can be justified by what they let us explain about known results suggests one possible example of such a scenario. He writes, "There might exist axioms so abundant in their verifiable consequences, shedding so much light upon a whole field, and yielding such powerful methods for solving problems ... that, no matter whether or not they are intrinsically necessary, they would have to be accepted at least in the same sense as any wellestablished physical theory" (1947).

For imagine a case where some proposed new axiom extending ZF set theory is known to be incompatible with some other axiom we are now moderately attracted to (e.g., some large cardinal axiom are known to be incompatible with the axiom of Choice [Kunen 2017]). And suppose it turned out that (analogous to what we seem to find regarding the access problem) the new axiom explained many "coincidences" as striking as the magic moonshine example above, and we (somehow) had reason to think that we could not satisfyingly explain these coincidences if the new axiom was false. In this case, we'd seem to have a very strong form of the kind of explanatory benefit Gödel endorses. So I think (if one is sympathetic to the Gödelian idea at all) it's quite conceivable that mathematicians could reject an antecedently attractive principle on the basis of intuitions about coincidence avoidance. However, I admit that it's hard to imagine what strong evidence that some axiom is necessary to explain some known regularity could look like. So I wouldn't be surprised if no such case can be found in the actual history of mathematics. 
problem for realist theories of mathematics, morals, and the like. I argued that various attempts to fix this problem by better specifying a reliability fact, $R$, which the realist is challenged to explain, fail.

I then suggested a reason for this failure: all such "single explanandum" accounts (in effect) get the logical structure of the access problem wrong. They attribute the access worrier an " $\exists \forall$ " intuition, that some particular apparent coincidence can't be explained by any realist account of human mathematical/moral accuracy (even a bump-pushing one). But what actually drives the access worry is a " $\forall \exists$ " intuition that (while it's usually easy to explain one mysterious predestined harmony by positing another) every realist account of human mathematical/moral accuracy would leave some mysterious coincidence unexplained.

Accordingly, I argued that access worriers would do better to stick closer to Field's informal statements. They should cash out access worries in terms of the realist's apparent commitment to some coincidence involving human accuracy about realist moral, mathematical, etc. facts. And they should reject demands for informative further analysis of what qualifies as a coincidence. Because of the good work which this notion of coincidence reduction already does in mathematics and the sciences, it is something to which all parties in debate are committed. Finally, I noted that we don't need to go beyond this unambitious way of formulating access worries in order to resolve debate about them.

Biography: Sharon Berry received her Ph.D. in Philosophy from Harvard and joined Oakland University after postdoctoral research at Australian National University and the Polonsky Academy in Jerusalem. Her primary areas of research are epistemology and the philosophy of mathematics. She has published papers on the Benacerraf problem, a priori knowledge, modal metaphysics, and potentialist set theory. 


\section{References}

Baker, Alan. 2009. "Mathematical Accidents and the End of Explanation." In New Waves in Philosophy of Mathematics, edited by Otávio Bueno and $\varnothing$ ystein Linnebo, 137-59. Basingstoke, UK: Palgrave Macmillan.

Benacerraf, Paul. 1973. “Mathematical Truth." Journal of Philosophy 70: 661-80.

Berry, Sharon. 2018. Not Companions in Guilt. Philosophical Studies 175 (9): 2285-308.

Berry, Sharon. Forthcoming. The residual access problem.

Bhogal, Harjit. Forthcoming. "Coincidences and the Grain of Explanation." Philosophy and Phenomenological Research.

Clarke-Doane, Justin. 2014. "Moral Epistemology: The Mathematics Analogy." Nous 48 (2): 238-55.

Clarke-Doane, Justin. 2017. "What Is the Benacerraf Problem?" In Truth, Objects, Infinity: New Perspectives on the Philosophy of Paul Benacerraf, 2nd ed., edited by Fabrice Pataut. Switz.:Springer International.

Donaldson, Thomas Mark Eden. 2014. “If There Were No Numbers, What Would You Think?” Thought: $A$ Journal of Philosophy 3 (4): 283-87.

Enoch, David. 2010. "The Epistemological Challenge to Metanormative Realism: How Best to Understand It, and How to Cope with It." Philosophical Studies 148 (3): 413-38.

Field, Hartry. 1980. Science without Numbers: A Defense of Nominalism. Princeton, NJ: Princeton University Press.

Field, Hartry. 1989. Realism, Mathematics, and Modality. Oxford: Blackwell.

Gödel, Kurt. 1947. “What Is Cantor's Continuum Problem?” In Kurt Gödel: Collected Works, Vol. II, edited by Solomon Feferman et al., 176-87. Oxford: Oxford University Press.

Goldman, Alvin, and Bob Beddor. 2016 (Winter). "Reliabilist Epistemology." In The Stanford Encyclopedia of Philosophy, edited by Edward N. Zalta.

Hellman, Geoffrey. 1994. Mathematics without Numbers. New York: Oxford University Press. Hirsch, Eli. 2010. Quantifier Variance and Realism: Essays in Metaontology. New York: Oxford University Press. 
Hume, David. 2007. An Enquiry Concerning Human Understanding and Other Writings. Cambridge: Cambridge University Press.

Kitcher, Philip. 1981. “Explanatory Unification.” Philosophy of Science 48 (4): 507-31.

Klarreich, Erica. 2017. Mathematicians Chase Moonshine's Shadow. In The Best Writing on Mathematics 2016, edited by Mircea Pitici. Princeton, NJ: Princeton University Press.

Kunen, Kenneth. 2017. Set Theory: An Introduction to Independence Proofs, Vol. 102. Amsterdam: Elsevier.

Lando, Tamar. 2016. Coincidence and Common Cause. Noûs 51 (1): 132-51.

Lange, Marc. 2010. “What Are Mathematical Coincidences (and Why Does It Matter)?” Mind 119 (474): 307.

Lewis, David K. 1986a. “Causal Explanation.” In Philosophical Papers, Vol. II, 214-40. New York: Oxford University Press.

Lewis, David K. 1986b. On the Plurality of Worlds. Malden, MA: Blackwell Publishers.

Linnebo, Øystein. 2006. “Epistemological Challenges to Mathematical Platonism." Philosophical Studies 129 (3): 545-74.

Nolan, Daniel. 1997. "Impossible Worlds: A Modest Approach." Notre Dame Journal of Formal Logic 38 (4): 535-72.

Wikipedia. "Planets Beyond Neptune." Accessed October 27, 2016. https://en.wikipedia.org/wiki/ Planets beyond Neptune.

Wright, Crispin. 1983. Frege's Conception of Numbers as Objects. Scotland: Aberdeen University Press. 